\title{
Chapter 17 \\ Biomaterials as Tendon and Ligament Substitutes: Current Developments
}

\author{
Mariana L. Santos, Márcia T. Rodrigues, Rui M.A. Domingues, \\ Rui Luís Reis and Manuela E. Gomes
}

\begin{abstract}
Tendon and ligament have specialized dynamic microenvironment characterized by a complex hierarchical extracellular matrix essential for tissue functionality, and responsible to be an instructive niche for resident cells. Among musculoskeletal diseases, tendon/ligament injuries often result in pain, substantial tissue morbidity, and disability, affecting athletes, active working people and elder population. This represents not only a major healthcare problem but it implies considerable social and economic hurdles. Current treatments are based on the replacement and/or augmentation of the damaged tissue with severe associated limitations. Thus, it is evident the clinical challenge and emergent need to recreate native tissue features and regenerate damaged tissues. In this context, the design and development of anisotropic bioengineered systems with potential to recapitulate the hierarchical architecture and organization of tendons and ligaments from nano to macro scale will be discussed in this chapter. Special attention will be given to the state-of-the-art fabrication techniques, namely spinning and electrochemical alignment techniques to address the demanding requirements for tendon substitutes, particularly concerning the importance of biomechanical and structural cues of these tissues. Moreover, the poor innate regeneration ability related to the low cellularity and vascularization of tendons and ligaments also anticipates the importance of cell based strategies, particularly on the stem cells role for the success of tissue engineered therapies. In summary, this chapter provides a general overview on tendon and ligaments physiology and current conventional treatments for injuries caused by trauma and/or disease. Moreover, this chapter presents tissue engineering approaches as an alternative to overcome the limitations of current therapies, focusing on the discussion about scaffolds design for tissue substitutes to meet the regenerative medicine challenges towards the functional restoration of damaged or degenerated tendon and ligament tissues.
\end{abstract}

M.L. Santos · M.T. Rodrigues · R.M.A. Domingues · R.L. Reis · M.E. Gomes $(\bowtie)$

3B's Research Group, Biomaterials, Biodegradables and Biomimetics, European Institute of Excellence on Tissue Engineering and Regenerative Medicine, Avepark-Parque de Ciência E Tecnologia, Zona Industrial Da Gandra, 4805-017 Barco GMR, Portugal e-mail: megomes@dep.uminho.pt

M.L. Santos · M.T. Rodrigues - R.M.A. Domingues - R.L. Reis · M.E. Gomes ICVS/3B's—PT Government Associate Laboratory, Braga, Guimarães, Portugal 


\subsection{Tendon/Ligament Physiology and Properties}

Ligaments and tendons are connective tissues formed by dense bands of collagenous fibers. The well-ordered arrangement of these fibers within the tissue confers highly anisotropic mechanical properties and parallel fiber arrangements from the nano to the microscale. Tendons connect muscle to bone, allowing movement by transmitting forces generated by the muscle to the bone, while ligaments connect bone to bone stabilizing movement when forces are applied. Therefore these tissues of the human body play important roles in musculoskeletal biomechanics, especially at the joint level [1-3].

Tendons and ligaments vary in size, shape, orientation and anatomical location, although, in general, they are characterized by the presence of an abundant extracellular (ECM) with a few cells, the tenocytes, dispersed in rows in between the collagen fibers. The major compound of tendon ECM is type I collagen representing $60-85 \%$ of the tendon dry weight. Type I collagen confers stiffness and strength to the tissue but other types of collagen exist in minor amounts, namely type III, V, X, XI, XII and XIV collagens. Type V collagen has been associated to type I collagen in the regulation of the collagen fibril diameter while type III collagen is functionalized in tendon repair. Type XII collagen is present in the surface of fibrils and bonds the fibrils with other matrix components such as decorin and fibromodulin $[4,5]$. Due to the similarities between tendon and ligament composition as well as the complementary functions they perform in joint movement, biomechanics and stability, they are often described as the same type of tissue in tissue engineering strategies.

Tendon architecture presents a unique hierarchical organization where collagen molecules assemble and form subunits of increasing diameter and complexity (Fig. 17.1). The highly aligned collagen fibers arranged in a longitudinal way and parallel to the mechanical axis confer high tensile strength to the structure of these tissues.

The mechanical role of tendons and ligaments is based on their visco-elasticity properties allowing these structures to regain the original shape after deformation, when the deformation load resultant from the application of an external force is removed. This phenomenon occurs because of the high degree of resilience of these tissues, characterized by the capacity to absorb and store energy within the elastic range (understretch) and release that energy (when load is removed) so the tissue matrix recoils back and restores its original shape. These tissues are also temperature sensitive, which affects the rate of creep (slow elongation). For an effective elongation, tendons and ligaments should be heated and subjected to a significant load over a long time period to produce creep. If the load is sudden or excessive, the elastic limits of tendon and ligament may be exceeded and the tissue enters the plastic domain (Fig. 17.2). In this domain, the original shape cannot be restored and the tissue is permanently deformed. If loading forces continue over the plastic domain, the tissue reaches the failure point, which results in fiber rupture (Fig. 17.2) [1-3]. 


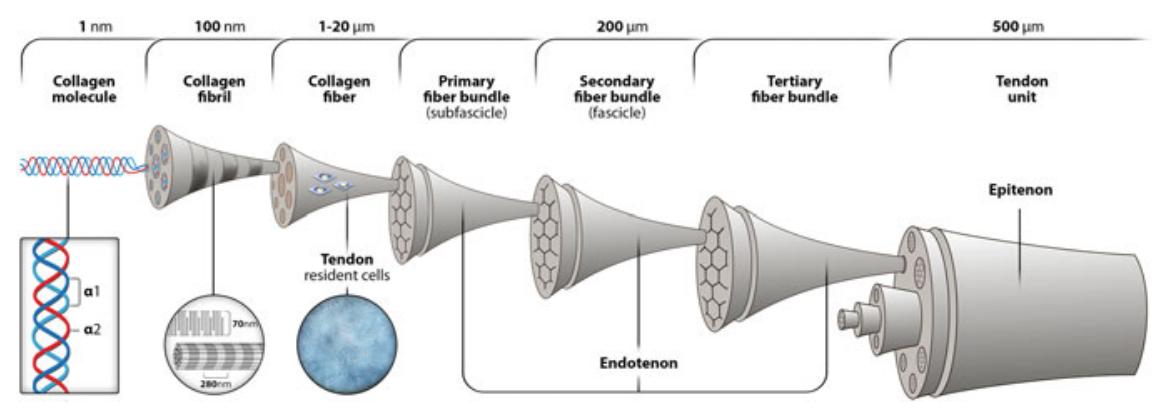

Fig. 17.1 Schematic representation of tendon/ligament architecture hierarchically organized into structural units. The tendon unit is composed of multiple fascicles of collagen fibers that result from the assembly of collagen fibers. These fibers are formed by fibrils that are formed by the aggregation of collagen molecules. This well-organized hierarchical structure from the nano to the microscale confers the anisotropic nature of tendons and ligaments and is responsible for the biomechanical properties of these tissues. Adapted from Ref. [6]. Copyright 2015, with permission of Springer

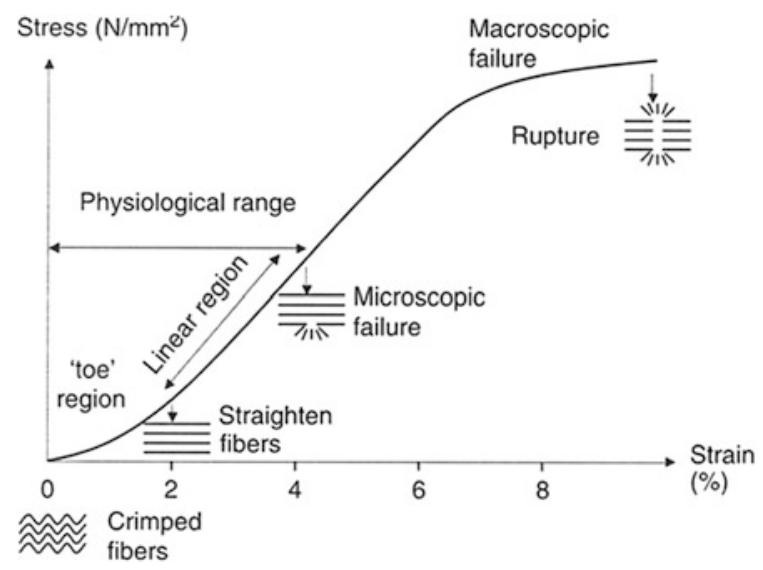

Fig. 17.2 Tendon stress-strain curve. The mechanism of tendon deformation expresses a nonlinear behavior consisting of a toe, linear, and yield regions in a stress-strain curve. Tissue fibers present a linear region until approximately $4 \%$ strain. After that value fibers have microscopic failures and rupture by $10 \%$ strain, showing macroscopic failure. Reprinted from Ref. [7]. Copyright 2006, with permission from Elsevier 
Table 17.1 Mechanical properties of tendon and ligaments of the knee

\begin{tabular}{l|l|l|l|l}
\hline Tissue & $\begin{array}{l}\text { Elastic modulus } \\
(\mathrm{MPa})\end{array}$ & $\begin{array}{l}\text { Ultimate tensile strength } \\
(\mathrm{MPa})\end{array}$ & $\begin{array}{l}\text { Ultimate strain } \\
(\%)\end{array}$ & References \\
\hline MCL & $332.3 \pm 58.3$ & $38.6 \pm 4.8$ & $17 \pm 2$ & {$[8,9]$} \\
\hline ACL & $65-447$ & $13-46$ & $15-44$ & {$[8,9]$} \\
\hline PSCL & $150-447$ & $30-36$ & $11-19$ & {$[8,9]$} \\
\hline LCL & $345 \pm 22.4$ & $36.4 \pm 2.5$ & $16 \pm 0.8$ & {$[10]$} \\
\hline
\end{tabular}

$A C L$ anterior cruciate ligament, $M C L$ medial collateral ligament, $P S C L$ posterior cruciate ligament, $L C L$ lateral collateral ligament

Furthermore, the strength response of tendons and ligaments under loading is determined by two main factors: size and shape of the tissue and speed of loading. The higher the number of oriented fibers in the direction of the loading the stronger with be the tissue, especially if they are wider and thicker [2]. The biomechanical properties of tendons and ligaments within the same joint vary significantly accordingly to their specific function. Knee joint is stabilized by four main ligaments: anterior cruciate ligament (ACL) which restrains anterior translation of the tibia relative to the femur, posterior cruciate ligament (PSCL) restraining posterior tibial displacement, medial collateral ligament (MCL) which restrains valgus angulation and lateral collateral ligament (LCL) with the function of restraining varus angulation. ACL presents the highest value of elastic modulus, ultimate tensile strength and ultimate strain (Table 17.1) among the described tissues evidencing more resistance to deformity when forces are applied to $[8,9]$.

\subsection{Tendon and Ligament Response to Injury and Regeneration}

Tendon and ligament injuries and associated diseases are a common problem and a leading cause of joint disability affecting athletes, active working people and the elder population worldwide. This problematic influences the quality of life of the affected population, once locomotion and local structure integrity are severely compromised.

Tendon and ligament lesions can occur through acute or chronic changes or a combination of both. Intrinsic factors as genetics, age, nutrition or misalignments are more often associated to chronic injuries while extrinsic factors namely pharmacological drug treatment or excessive or absence of mechanical loading have been related to acute injuries. Acute injuries are more frequently related to sports injuries, being the ACL the most affected to rupture in the knee joint $[9,11]$. 
Repetitive trauma, traumatic or chemically induced injury and disuse have been described as major causes for pathogenic conditions. The healing or fail to heal capacity of tendon and ligament has a dependent correlation with intrinsic and extrinsic factors. A failed mechanism will induce molecular and histological changes affecting cellularity, tissue extracellular matrix and vascularity resulting in mechanical weakness, pain and eventual tear or complete rupture [12].

Tissue injury, characterized by three main stages (inflammation, necrosis and pain), can progress toward repair or regeneration. In tissue repair scar tissue will be formed with inflammatory cell infiltration, fibroplasia, disorderly collagen disposition and consequently, impaired mechanical properties. On the other hand, if regeneration occurs the tissue to heal will present few inflammatory cells, absence of fibroplasia, orderly collagen deposition and consequently, restoration of mechanical properties and absence of fibrotic tissue. Therefore regeneration is the desired evolution of injury for a complete regain of tissue functionality.

\subsection{Current Conventional Treatments}

Current available management of tendon and ligament injuries rely on conservative treatments and or surgically interventions (Table 17.2), which depend on the physio-anatomy of tissue, symptoms and clinical findings on the type and severity of the damage. Despite treatments, the mid to long term outcomes are not completely successful and tendon and ligament injuries will likely progress to nearby tissues and ultimately evolve into mild to severe forms of osteoarthritis (OA).

Because of the limitations and frequent failure of nonsurgical approaches, surgery remains the treatment of choice, especially for athletes suffering from ligament injuries, who want to remain competitively active.

Despite clinical advances and knowledge on surgical management, the replacement of damaged tissue with tissue grafting is still a gold standard despite the morbidity and functional disability of donor tissue that may have severe consequences in the long term that include pain, instability, loss of mechanical competence and degeneration of both tissue and joint.

The most commonly used grafts in anterior cruciate ligament (ACL) reconstruction are the hamstring tendon and patellar tendon. Hamstring and bone-patellar tendon-bone autografts are described to allow approximately $50 \%$ of patients to return to their pre-injury sporting activity level. Hamstring grafts lead to better preservation of extension, higher patient-reported outcome scores, and less radiographic evidence of OA [17]. A recent study also reported a prevalence of patellofemoral OA in $26 \%$ of the patients 12 years after ACL reconstruction function [18]. The prevalence of patellofemoral OA for the contralateral knee was 
Table 17.2 Conventional treatments and associated limitations in the management of tendon and ligament injuries

\begin{tabular}{|c|c|c|c|c|}
\hline $\begin{array}{l}\text { Type of } \\
\text { treatment }\end{array}$ & $\begin{array}{l}\text { Aim of the } \\
\text { treatment }\end{array}$ & Approach & Limitation & Ref. \\
\hline $\begin{array}{l}\text { Conservative } \\
\text { management }\end{array}$ & $\begin{array}{l}\text { - Control of pain } \\
\text { - Reduction of } \\
\text { inflammation } \\
\text { and swelling }\end{array}$ & $\begin{array}{l}\text { - Rest } \\
\text { - Cryotherapy } \\
\text { - Injection therapy (corticosteroids, } \\
\text { sclerotherapy and hemoderivatives) } \\
\text { - Orthontics } \\
\text { - Continuous passive motion } \\
\text { - Restrictive bracing } \\
\text { - Ultrasounds } \\
\text { - Laser treatment } \\
\text { - Electrotherapy } \\
\text { - Exercises at strengthening and balance }\end{array}$ & $\begin{array}{l}\text { - Initial phase of damage } \\
\text { - Limited success } \\
\text { - Fail to regenerate tissue } \\
\text { - Risk of disease/injury } \\
\text { progression }\end{array}$ & $\begin{array}{l}{[13-} \\
15]\end{array}$ \\
\hline $\begin{array}{l}\text { Surgery } \\
\text { intervention }\end{array}$ & $\begin{array}{l}\text { - Reduce the } \\
\text { symptoms } \\
\text { - Stabilize and } \\
\text { improve } \\
\text { articular } \\
\text { function }\end{array}$ & $\begin{array}{l}\text { - Removal of damaged tissue (areas of failed } \\
\text { healing, fibrosis and pathological nerve } \\
\text { ingrowth) } \\
\text { - Application of augmentation devices or } \\
\text { patches or recurring to auto and allo-grafts to } \\
\text { replace damaged tissue } \\
\text { - Long rehabilitation }\end{array}$ & $\begin{array}{l}\text { - Instability } \\
\text { - Increased risk of failure } \\
\text { and recurrence } \\
\text { - Formation of scar tissue } \\
\text { and or adhesions } \\
\text { - Fail to regenerate tissue } \\
\text { - Loss of tissue } \\
\text { mechano-competence } \\
\text { associated to } \\
\text { functionality } \\
\text { - Mechanical mismatch } \\
\text { and tissue laxity } \\
\text { - Risk of nerve damage } \\
\text { and infection } \\
\text { - Expiration date } \\
\text { - No protection against } \\
\text { long term degenerative } \\
\text { changes } \\
\text { In autografts: } \\
\text { - Morbidity and functional } \\
\text { disability at the } \\
\text { harvesting site } \\
\text { - Poor tissue } \\
\text { integration/non-anatomic } \\
\text { placement } \\
\text { - Graft impingement or } \\
\text { tension } \\
\text { In allografts: } \\
\text { - Need for } \\
\text { immunosuppressive } \\
\text { drugs to avoid tissue } \\
\text { rejection } \\
\text { - Poor tissue } \\
\text { integration/non-anatomic } \\
\text { placement } \\
\text { - Risk of pathogen } \\
\text { transmission } \\
\text { - Graft impingement or } \\
\text { tension }\end{array}$ & $\begin{array}{l}{[13-} \\
16]\end{array}$ \\
\hline
\end{tabular}

$6 \%$, but only $2.5 \%$ for uninjured contralateral knee. Significant associations were also found between patellofemoral OA and increased age, tibiofemoral OA and impaired function [18]. 
Other approaches involving artificial augmentation devices are also available for tissue reconstruction [19]. These include commercially available devices as LARS ${ }^{\mathrm{TM}}$, Leeds-Keio, Kennedy ligament augmentation device, Dacron, Gore-Tex and Trevira [20] being LARS one of the mostly used for ACL and PSCL. They offer advantages over tissue grafts by avoiding donor tissue morbidity and providing improved knee stability [19] and full weight bearing. Nevertheless they also have limitations and several complications have been associated to the long term follow up with artificial devices, namely, mechanical failure or mechanical mismatch with native tissues, synovitis, chronic effusions, recurrent instability and early knee OA.

Although these systems have been used for decades now, their outcomes are still controversial. Some studies with a 10 year follow up refer that the LARS ${ }^{\mathrm{TM}}$ system should not be suggested as a potential graft for primary reconstruction of the ACL [21] while others validate their application. Moreover, special indications have been described in literature for the effective and safe use of some of these devices [21].

Despite the potential and interest generated using biological augmentation for tendon and ligament reconstruction, surgeons do not seem convinced of their bio-mimicry benefits for the knee joint and preferentially choose artificial over biological devices. Biological augmentation is often mediated by decellularized mammalian-derived tissues, mainly from human $\left(\right.$ GraftJacket $\left.^{\circledR}\right)$, porcine $\left(\right.$ Restore $\left.^{\mathrm{TM}}\right)$, equine $\left(\right.$ OrthADAPT $\left.^{\circledR}\right)$ or bovine (TissueMend ${ }^{\circledR}$ ) origin [22]. The risk of immune-rejection and of zoonose transmission together with the lack of publications in recent years, limits the knowledge and clinical outcomes of patients treated with these matrices.

Tendon morphology and functionality are intrinsically associated and function depends on the highly organized hierarchy of parallel, crosslinked fibrils of collagen assembled from nano to macro structures. Partial or total loss of tendon and ligament functionality is mainly caused by a poor alignment of collagen fibrils in scar tissue, resulting in significant mechanical limitation of repaired tendons that never match the properties of healthy non-injured tissue. Thus, the creation of artificial 3D highly sophisticated and complex systems that recapitulate this hierarchical and anisotropic architecture to support a complete regeneration of damaged tissues while remaining mechanically competent is a challenge to overcome by tissue engineering technologies.

\subsection{Tissue Engineering Strategies for Tendon and Ligament Regeneration}

\subsubsection{Tissue Engineering and Regeneration}

Regeneration represents one of the most important biological processes, assisting the renewal and remodeling of tissues and organs which have suffered physical damage or injury. With regeneration the normal structure and function of the 
tissue/organ are completely restored into a functional level and homeostasis is reestablished. On the other hand, this does not occur in tissue repair, a failed attempt to regenerate, resulting in the synthesis of disorganized fibrotic tissue with inferior properties to the original healthy tissue [23]. Thus, tissue engineering and regenerative medicine proposes alternative approaches combining living agents, the cells, with 3D structures to mimic the biophysical and chemical cues of native extracellular matrix, and/or bioactive molecules to biochemically stimulate cells and the tissue milieu, to meet the demanding requirements of tissue regeneration.

\subsubsection{Cell-Based Strategies for Tendon and Ligament Tissues}

The hypocellular and hypovascular nature of tendons and ligaments comparatively to other tissues has been associated to a very limited natural healing capacity with significant drawbacks for a successful regeneration, especially when severe injuries occur. Moreover, failure to regenerate increases the risk for progression of associated diseases into nearby tissues, inflicting more pain and degeneration to the already injured joint $[5,24]$. Thus, it is not surprising that some potential regenerative approaches for tendon and ligament focus on cell based strategies to overcome these limitations and accelerate a tissue regenerative response.

Tendon and ligament resident cells are an obvious choice [25] since these cells are harvested from the target tissue and an eventual level of epigenetic memory could match the desired cell response to meet regeneration in damaged tendons or ligaments. In $2007 \mathrm{Bi}$ and co-workers discovered a tendon stem/progenitor cell population with functionally attractive features including universal stem cell characteristics such as clonogenicity, multipotency and self-renewal capacity, and with the capability to generate a tendon-like tissue after in vitro expansion and in vivo transplantation [26]. Although isolating autologous cells from tendons and ligaments is a feasible process, the harvesting of resident cells, even in limited number, is not the most adequate option, as it may interfere with donor tissue homeostasis, causing severe tissue morbidity. Cells harvested from a different donor are a valid but not so desirable alternative, since tissue supply is limited and there is an associated risk of rejection or disease transmission.

Pluripotent embryonic stem cells (ESCs) are an alternative source to tendon cells, whose potential for the treatment of tendon injuries has been demonstrated in a patellar defect of a rat model [27], resulting in improved mechanical and structural properties without teratoma formation. Nevertheless, the ethical issues associated to the manipulation of human embryos, and the risk of tumor formation post implantation limits advances in human ESC knowledge and prevents new insights for regenerative medicine.

Induced pluripotent stem cells (iPSCs) technology also presents value for tendon and ligament regeneration, as iPSCs can be reprogrammed into a wide range of cell 
types providing an inexhaustible source of autologous cells without the ethical considerations of ESCs but still resembling and sharing some ESC characteristics. A recent study reported that a treatment with iPSCs derived from neural crest stem cells significantly enhanced tendon healing in a window defect of a rat patellar tendon with improvement in matrix synthesis and mechanical properties [28]. Despite interesting outcomes and a promising future for clinical applications, it is necessary to improve the production efficiency of human iPSCs and assess human safety application for cell therapy.

Adult tissues may also be interesting alternatives as stem cell sources. It is widely described that practically all tissues in the human body have a stem cell population that participates in the endogenous regeneration of the tissue. The role of these stem cells is mediated by the release of trophic factors that influence tissue milieu. Adult stem cells are not pluripotent as ESCs but can commit and differentiate into several tissue lineages and have a high self-renewal capacity.

Bone marrow stem cells are the most studied stem cells of adult origin and were shown to have tenogenic differentiation potential [29, 30]. Furthermore, human bone marrow mesenchymal stem cells (MSCs) supported tendon healing when implanted into artificially induced tendinitis in rat Achilles tendon, promoting neovascularization and produced larger deposits of type I collagen and type III collagen and better organization of the extracellular matrix [31]. No tumor formation or excessive inflammatory reaction was locally detected at the rat tendon [31].

Adipose tissue [32] and amniotic fluid [32] have been also reported to be promising for tendon repair, having the ability to commit into a tenogenic phenotype as measured by increased genetic and protein expression of tendon related markers, namely type I and III collagen, decorin, tenascin C and scleraxis under supplemented culture medium. In comparison to other adult stem cell sources, adipose tissue offers a more abundant source and less invasive procedures for harvesting stem cells with immunomodulatory properties and long-term genetic stability.

Adipose tissue-derived MSCs were applied to the treatment of induced tendinitis of the superficial digital flexor tendon in the horse [33]. The lesions that received treatment with these cells presented a more organized and uniform tissue repair as compared with the control limb, including less inflammatory infiltrate, greater parallel arrangement of the fibers, larger extracellular matrix deposits, and greater type I collagen expression [33].

Despite the growing knowledge on regeneration mediated by stem cells and the fact that bone marrow stem cells have already found a clinical niche in cell therapies for the treatment of several (non-tendon/ligament) diseases, stem cell therapies for tendon and ligament require further research in order to understand the mechanisms of regeneration, recapitulate them in vitro and translate the appropriate stimuli in a spatial-temporal manner toward successful cell-based therapeutic tools. The still limited knowledge about the tenogenic process and associated markers together with the lack of standardization of biochemical cocktails to induce in vitro tenogenesis are holding back the understanding and recapitulation of tissue 
regeneration. However, it is expected that these drawbacks will be overcome in the next few years and will bring significant insights in therapeutics toward tendon and ligament tissues.

\subsubsection{Design and Fabrication of 3D Sophisticated Scaffolds}

As mentioned above, the complex hierarchical ECM is essential for tendon and ligament functionality and responsible to be an instructive microenvironment for resident tendon cells.

A key challenge in tendon and ligament TE is exactly the recreation of 3D scaffolding biomaterials that can mimic this unique architecture and support tissue regeneration while remaining mechanically competent [6, 7].

Being mechano-sensitive and mechano-responsive tissues, the cell response may be assisted and guided by 3D structures that would recreate tendon microenvironment with specific topographical and biophysical cues such as the substrate geometry and topography of fiber based scaffolds. The incorporation of growth factors (GFs) and other bioactive molecules within a 3D scaffold can also improve the biofunctionality of the system, once GFs were shown to play a crucial part in tendon and ligament repair [34-41].

However, the development of tendon/ligament scaffolds is a nontrivial issue as they should match the mechanical properties of the targeted tissue in order to allow appropriate functionality, but progressively degrade over time at a rate matching tissue regeneration while preserving the overall construct tensile properties and reduce the risk of premature rupture. Thus, a suitable scaffold should be tailored considering the properties of the biomaterials they are produced from, the scaffold design and architecture as well as the processing technique [42].

Scaffold biomaterials can be synthetic, natural based or a combination of both. Synthetic polymers are known for their higher processability being more versatile in fitting a wide range of properties and structural features, while natural polymers, such as alginate, chitosan, hyaluronic acid are obtained from renewable and abundant sources and may be biodegraded by enzymes naturally present in the body. Moreover, the fact that the biological and chemical properties of the latter share similarities to living tissues, in particular to the extracellular matrix, can be an advantageous parameter for cellular recognition in TE strategies.

Several potential biomaterials for the development of tendon or ligament scaffolds and associated advantages are summarized in Table 17.3.

Aligned fibrous materials have been among the preferred options as potential scaffolds in tendons and ligaments [64-66] mainly due to the linear and fibrillar organization of collagen molecules into fibrils, fiber bundles, fascicles and tendon units. These materials can be obtained through different fiber fabrication technologies, but in the past few years electrospinning [64, 65] and electrochemical alignment technique [46, 67] combined with textile techniques have been in the 
Table 17.3 Examples of scaffold biomaterials that have been studied for tendon and ligament TE strategies

\begin{tabular}{|c|c|c|c|c|}
\hline Origin & Biomaterial & $\begin{array}{l}\text { Processing } \\
\text { method }\end{array}$ & Advantages & References \\
\hline \multirow[t]{3}{*}{ Natural } & Collagen & $\begin{array}{l}\text { Wet-spinning } \\
\text { Electrochemical } \\
\text { alignment }\end{array}$ & $\begin{array}{l}\text { Reasonable } \\
\text { mechanical properties } \\
\text { Relatively slow rate of } \\
\text { degradation } \\
\text { Main component of } \\
\text { T/L }\end{array}$ & [43-46] \\
\hline & Silk & $\begin{array}{l}\text { Electrospinning } \\
\text { Knitting }\end{array}$ & $\begin{array}{l}\text { Good mechanical } \\
\text { properties } \\
\text { Slow rate of } \\
\text { biodegradation }\end{array}$ & $\begin{array}{l}{[39,47-} \\
51]\end{array}$ \\
\hline & Alginate/CHT & Wet-spinning & $\begin{array}{l}\text { Provide a proper } \\
\text { substrate for fibroblast } \\
\text { growth with dense } \\
\text { type I collagen } \\
\text { production }\end{array}$ & [52] \\
\hline \multirow[t]{4}{*}{ Synthetic } & PLGA & Electrospinning & \multirow{4}{*}{$\begin{array}{l}\text { Easier to process } \\
\text { through different } \\
\text { techniques } \\
\text { Large scale production } \\
\text { with lower cost } \\
\text { Higher mechanical } \\
\text { properties comparing } \\
\text { with natural polymers }\end{array}$} & {$[39,53]$} \\
\hline & PLLA & \multirow[t]{2}{*}{ Melt-spinning } & & [54-57] \\
\hline & PGA & & & [55] \\
\hline & PCL & $\begin{array}{l}\text { Freeze drying } \\
\text { Electrospinning }\end{array}$ & & {$[58,59]$} \\
\hline \multirow[t]{3}{*}{ Synthetic/naturals } & PLCL/Collagen & \multirow[t]{3}{*}{ Electrospinning } & \multirow{3}{*}{$\begin{array}{l}\text { Combination of the } \\
\text { best properties of } \\
\text { natural and synthetic } \\
\text { polymers }\end{array}$} & {$[60,61]$} \\
\hline & PCL/CHT & & & {$[62]$} \\
\hline & PCL/CHT/CNC & & & [63] \\
\hline
\end{tabular}

T/L tendon/ligament, PLGA poly(lactic-co-glycolic acid), PLLA poly-L-lactic acid, PGA poly (glycolic acid), $P C L$ polycaprolactone, $P L C L$ poly(L-lactide-co- $\varepsilon$-caprolactone), $C H T$ chitosan, CNC cellulose nanocrystals

development forefront of hierarchical scaffolds for the proposed TE applications. These strategies have been showing promising results in this field and will be discussed in more detail in the following sections.

\subsubsection{Electrospinning}

Electrospinning produces long continuous fibers with controlled diameter from nanometers to microns. The advantage of electrospinning comparing with other conventional techniques is that the produced fibrilar systems better mimic the nanoscale morphological structure of tendon and ligament ECM, in order to provide the topographical cues and promote cells contact guidance, increasing the potential for regeneration. 
Following this strategy it is possible to artificially reproduce the characteristic anisotropic alignment of collagen fiber bundles in these tissues [68, 69].

This technique also enables the production of fibers from different polymers including those from natural origin, such as collagen, chitosan, hyaluronic acid and silk fibroin; or synthetic, for example poly( $\varepsilon$-caprolactone) (PCL), poly(glycolide) (PLGA), poly(L-lactide) (PLA). Using natural and synthetic polymeric blends it is possible to combine in a single system the adequate mechanical properties from synthetics and the favorable biological response of cells/tissue from natural based biomaterials aiming to mimicking the natural ECM of tendon and ligament [53, 70, 71]. These include e.g., poly(L-lactide-co- $\varepsilon$-caprolactone)/collagen [60, 61] or poly$\varepsilon$-caprolactone/chitosan (PCL/CHT) $[62,72]$ aligned nanofibrous scaffolds.

Several systems have been tested to produce aligned nanofiber mats, but high speed rotating collectors forcing to an aligned nanofiber deposition is usually the preferred strategy to produce T/L nanofiber scaffolds [65].

The resulting nano/microtopography of the scaffolds fabricated through this technique has proven advantageous. Anisotropically aligned nanofibrous scaffolds provide tendon biomimetic cues that induced cell alignment through contact guidance mechanisms, resulting in proved impact on cell alignment along the nanofiber aligned axis, but also over stem cells differentiation, phenotype maintenance as well as matrix deposition, while recreating the anisotropic mechanical behavior of tendon tissues [24, 53, 62, 71, 73, 74]. Moreover, this type of well-aligned fiber scaffolds has recently demonstrated to enable the multistep tenogenic differentiation of hiPSCs in vitro and the resulting tissue engineered constructs promoted tendon repair in vivo in a rat Achilles tendon model [66]. The tenogenic commitment of these cells was assigned to the activation of the mechanic-signal pathway resulting from the cytoskeletal rearrangement induced by the scaffold's topography [66].

Recreating the characteristic non-linear tri-phasic deformation behavior of tendons and ligaments is also a very important feature to consider. The toe region under low deformation (typically between 0-2\% strain), which results from the uncramping of collagen fibrils, is important in tendon/ligament biomechanics as shock-absorbing feature to prevent tissue damage. Recent studies developed crimped electrospun scaffolds [75-77]. The crimped pattern on electrospun fiber mats provide a closer mimic of the nonlinear biomechanical behavior of collagen fibrils, which facilitate nonlinear stiffening of the tissue under increasing tensile strains [75]. Furthermore, tendon cells cultured on crimped nanofibers showed a higher level of tolerance toward externally applied strain than those cultured on the straight nanofibers, suggesting that the crimping feature in nanofiber-based scaffolds has a high potential for tendon and soft connective tissue repair.

Considering the anatomic load-bearing function of tendon and ligaments, the tensile behavior of the proposed scaffolds are also critical parameters. Scaffold's design for tendon/ligament applications should match the mechanical properties of native tissues, not only to minimize stress-shielding effects that may lead to disorganized tissue growth, but also to provide a temporary replacement for immediate 
function with reduced probability of premature rupture after repair, while allowing tissue regeneration over time [78].

The tensile properties reported for native tendons and ligaments are in the range of 5-100 MPa of ultimate tensile strength and 20-1200 MPa of Young's modulus, while strain at failure varies much less and typically fall in the range of 10-15\% [79]. A recurrent concern about the relevance of electrospun scaffolds in tendon/ligament TE strategies is related with their reported limited mechanical performance. Electrospun scaffolds have been preferentially fabricated based on semi-crystalline biodegradable polyesters and their tensile mechanical properties generally range from 1 to $25 \mathrm{MPa}$ in ultimate tensile strength and 1-350 $\mathrm{MPa}$ in elastic modulus [14]. While it may be sufficient when applied in comparatively low tensile demanding tendon/ligament tissues such as those from human shoulder, they are not satisfactory when targeting high tensile demanding tissues such as patellar, Achilles tendons or ACL. Our research group has recently addressed this issue by reinforcing tendon/ligament fibrous scaffolds made of a natural/synthetic polymer blend of PCL/CHT with the incorporation of cellulose nanocrystals (CNC) which are bioderived nanofillers [63]. The incorporation of low CNC contents (up to $3 \mathrm{wt}$ $\%$ ) into PCL/CHT polymer blend to form nanofiber bundles had a significant toughening effect (increased $132 \%$ the Young's modulus and $83 \%$ ultimate tensile strength without significantly affecting strain at failure). Moreover, this reinforcement was achieved while maintaining the structural cues for the superior biological performance (Fig. 17.3). This may thus be a suitable strategy to explore in order to fabricate tendon/ligament mimetic nanofibrous structures balanced with appropriate mechanical performance and expand their potential of applications in this field.

Nonetheless, electrospinning typically produces $2 \mathrm{D}$ fiber mats, restricting their further processing into higher hierarchical 3D structures. Thus, scaffolds are limited in terms of dimensions, handling and load-bearing capacity. Different strategies have been devised in recent years [80], including rolling [81] or twisting [82] pre-cut sections from aligned nanofiber to produce nanofiber bundles, or their assembly into higher 3D hierarchical structures through standard textile such as e.g. weaving or braiding $[24,74]$. These strategies allow producing aligned nanofiber scaffolds of relevant dimensions, while enabling to tune their general mechanical properties (stiffness, strength, strain and maximum load) to mimic the typical tri-phasic biomechanical behavior of tendons and ligaments by artificially recreating the characteristic toe region in a load-displacement curve.

However, these fabrication strategies are less practical in terms of clinical translation, as well as on their scale up production and standardization because they are based on the typical rotating collection drums or wheels which produce a $2 \mathrm{D}$ nanofiber sheet or bundle of limited dimensions. Therefore, the production of continuous electrospun nanofiber yarns as mimicry of tendon fascicles is suitable for further assembling into higher hierarchical 3D structures through standard textile techniques and devices would be a significant breakthrough in T/L TE. Several strategies for their production have been developed in the past few years [65] and recently Mouthuy et al. devised an automated method that enables the manufacture of continuous electrospun filaments with the potential to be integrated 

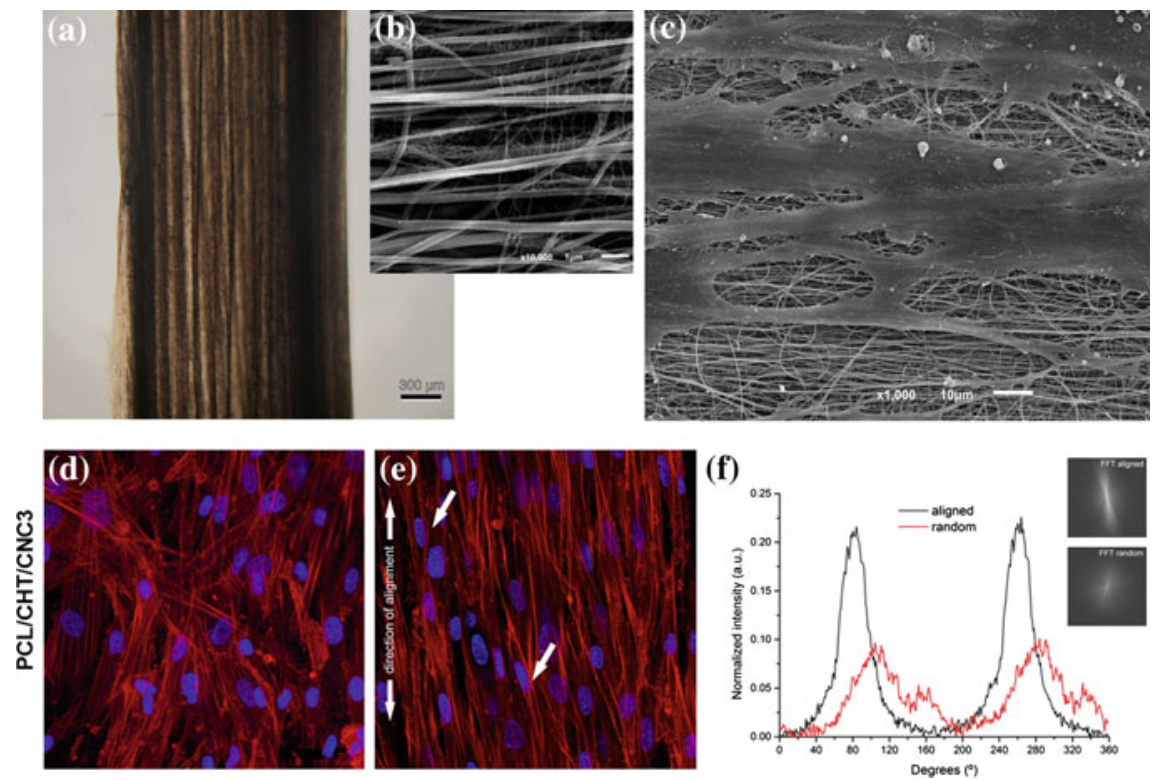

Fig. 17.3 Optical (a) and high magnification SEM (b) micrographs of aligned nanofiber bundles of PCL/CHT/CNC3. c SEM micrographs of hTDCs seeded on PCL/CHT/CNC3 nanofibrous scaffolds with aligned topography after 10 days of culture. Confocal microscopy micrographs of the hTDCs seeded on PCL/CHT/CNC3 nanofibrous scaffolds with random (d) and aligned (e) topography (blue nuclei stained with DAPI; red actin filaments stained with rhodamine-conjugated phalloidin). f Respective 2D FFT frequency plots (insets) and normalized radial intensity plotted against the angle of rotation for hTDCs cultured on random and aligned nanofibers. Scale bar $300 \mu \mathrm{m}$ (a), $1 \mu \mathrm{m}$ (b), $10 \mu \mathrm{m}$ (c). Reprinted from Ref. [63]. Copyright 2016 WILEY VCH Verlag GmbH \& Co. KGaA, Weinheim. With permission from John Wiley and Sons

into existing textile production lines $[65,83]$. In practice the proposed technology is a multistep process (Fig. 17.4) relying on the use of a wire guide to collect a nanofiber mesh which is then detached as a long and continuous thread, the thread is drawn to align the nanofibers and then is twisted to create multifilament yarns.

This concept, the first of his kind, is still in the early stages of development and lack optimization. However, further developments are expected in coming years on biotextile scaffolds based in continuous nanofiber yarns for $\mathrm{T} / \mathrm{L} \mathrm{TE}$.

In a different strategy to produce 3D hierarchical scaffolds for T/L TE, Yang et al. recently proposed a multilayered fiber-hydrogel composite approach [84]. The concept consisted in simultaneously co-electrospin PCL and methacrylated gelatin using the typical rotating collection drum. The $2 \mathrm{D}$ fiber mat sheets are wet with photoinitiator solution and then photocrosslinked to produce the fiber-hydrogel composite scaffolds. Stacking multiple sheets prior to photocrosslinking allows the production of multilayered scaffolds as well as the encapsulation of cells, if desired, within layers. Although the results support that a combination of nanofibrous 

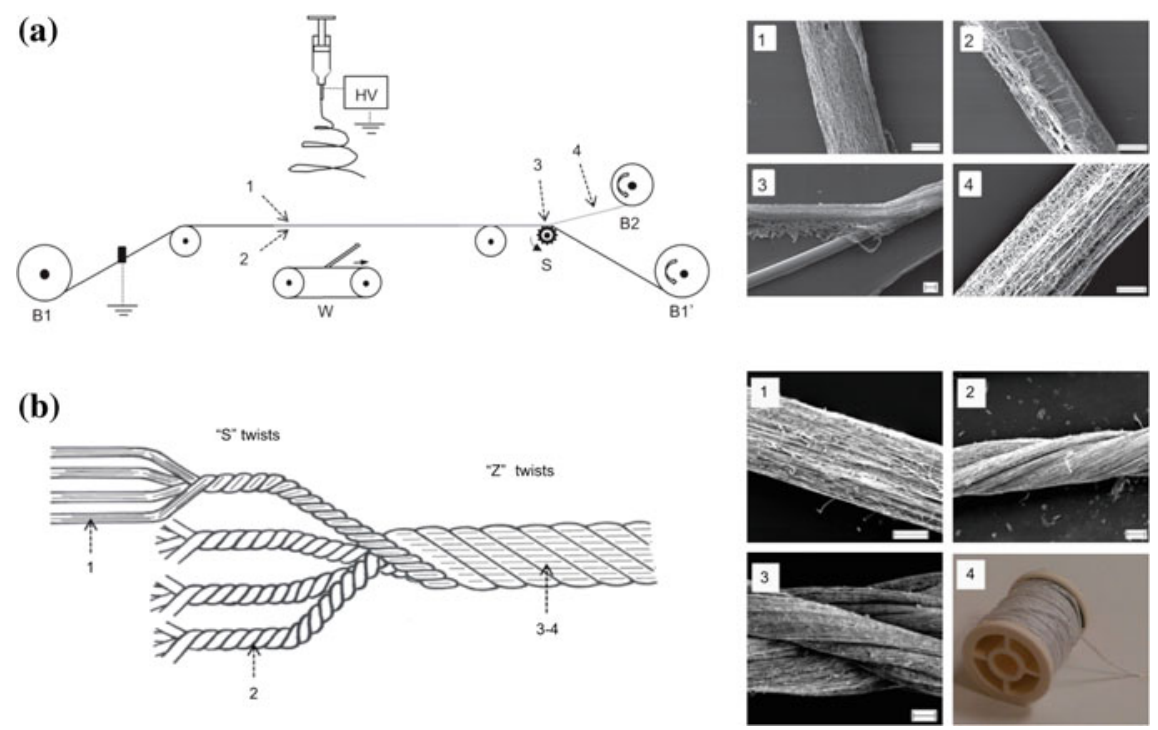

Fig. 17.4 Method used for the fabrication of continuous electrospun filaments and multi filament nanofiber yarns. a Sketch of the manufacturing process. The method consisted in spinning the polydioxanone (PDO) fibres on a stainless steel wire progressing at a speed of $0.6 \mathrm{~mm} \mathrm{~s}^{-1}$ underneath the electrospinning nozzle ( $B 1$ wire supply, $B 1^{\prime}$ wire collection, $B 2$ electrospun filament collection, $S$ cutter wheel, $W$ wiper). The electrospun material was then separated from the wire in the form of a continuous filament. (1-4) Scanning electron microscope images taken at different positions in the process. Fibers are mostly collected on the side of the wire exposed to the electrospinning jet (1) compared to the hidden side, (2) the mesh can be separated from the wire, (3) as one continuous thread of randomly oriented submicrofibres (4). b The stretched filaments (1) were assembled into 4-plied yarns by manually twisting four filaments together in a right-hand direction (' $\mathrm{S}$ ' turn) at 400 twists/m. (2) Four of these were then twisted together in a left-hand direction (' $\mathrm{Z}$ ' turn) at 200 twists/m to fabricate a cord yarn $(3,4)$. Adapted from Ref. [83] with permission

structures and photocrosslinked hydrogels may closer mimic the $\mathrm{T} / \mathrm{L}$ structure, providing the aligned topographical cues and contributing for the tenogenic differentiation of hASCs [84], the final mechanical properties are far from ideal (ultimate tensile strength of $1.45 \pm 0.19 \mathrm{MPa}$ ), which restricts their potential application in this field.

\subsubsection{Electrochemical Alignment Technique}

The so called electrochemical alignment technique is an interesting strategy that allows producing anisotropically aligned collagen bundles through a process based on the $\mathrm{pH}$ gradient created between two parallel electrodes [46, 67, 85]. This strategy was firstly proposed for TE of connective tissues by Akkus group [86], that have been developing this technique, culminating in a recent proposed system for 
the production of continuous electrochemically aligned collagen (ELAC) threads [67].

Their pioneer study has shown that the ELAC threads exhibit a collagen fibrillar organization that mimics the packing density, alignment and strength of T/L tissues [86]. Following studies evaluated the effect of several processing parameters in the mechanical and structural properties of ELAC threads, such as buffer concentration and incubation time [87], crosslinking degree (with genipin) [88] and addition of proteoglycan (decorin) to the collagen matrix [89]. Ultimately, the optimization resulted in the improvement of the of ELACs wet ultimate tensile strength to the range of 80-110 MPa, elastic modulus of $600-900 \mathrm{MPa}$ and strain of 10-15\%, which approximates the values for several native tendons.

Gurkan et al. compared the ability of tendon-derived fibroblasts (TDFs) and bone marrow stromal cells (MSCs) to migrate and populate single and braided ELAC threads crosslinked with genipin [46]. The results support the non-cytotoxicity of crosslinked ELACs and that in vitro both cell types colonize and migrate over ELAC threads more successfully than in singles threads [46]. It was also demonstrated that different crosslinking degrees and threads coagulation treatments have an impact over hMSCs adhesion and proliferation, probably resulting from the different threads stiffness [88]. Moreover, the anisotropically aligned topography of the genipin crosslinked ELAC threads proved to promote tenogenic differentiation of hMSCs [90] and ELAC braided scaffolds showed in vivo biocompatibility and biodegradability after 8 months in a rabbit patellar tendon model [91].

Recently Younesi et al. developed a custom made rotating electrode electrochemical alignment device able to produce ELAC threads in a continuous mode [67]. Applying biotextile techniques, woven 3D-biotextile scaffold were fabricated with these threads (Fig. 17.5A). The 3D woven structure not only mimics the hierarchical structure and non-linear tensile behavior of native tendons, as MSCs seeded on the scaffold also express increased tendon specific markers when compared to randomly oriented collagen gels. This 3D-biotextile scaffold woven purely from collagen has a remarkable high porosity $(80 \%)$ which promotes cell seeding across the bioscaffold (Fig. 17.5B), while the anisotropically aligned substrate texture topographically stimulates tenogenesis [67].

These woven 3D-biotextile scaffolds were tested to span a gap defect between the infraspinatus muscle and humerus in rabbit [92]. Although the main objective of the study was to develop the suturing scheme for grafting the scaffold, it was shown that the graft repair was able to withstand similar load as the direct tendon reattachment, thus demonstrating the potential of this system as a full load-bearing construct for segmental defects.

Overall, the previously described outcomes from the in vitro and in vivo studies on ELAC threads scaffolds are encouraging and promising for T/L TE applications. However, it is also important to refer that, considering the basic principles of this fabrication technology, there might be a particular limitation in terms of polymer matrix option. In fact, with the exception of one study [89], scaffolds have been exclusively based on type I collagen. Also, the performance of ELAC based 
A
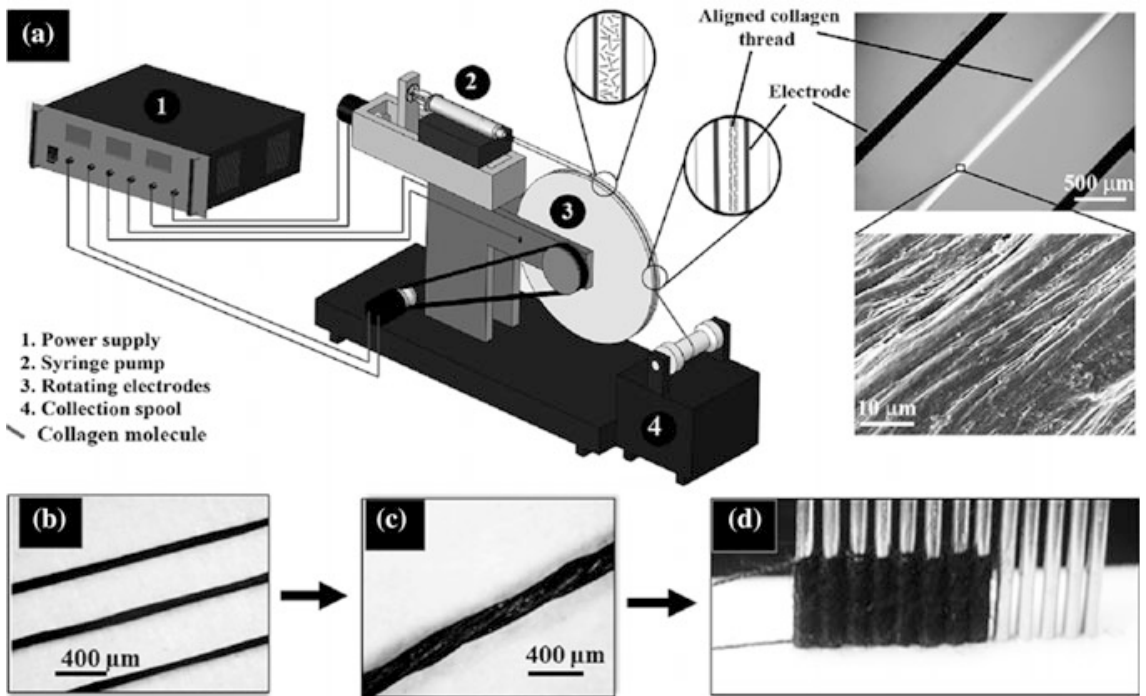

(ब) | || || || ||

ELAC threads

ELAC - Yarn (triple thread)

(f)

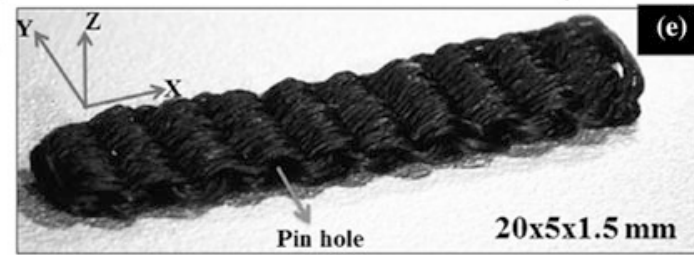

Morphologically consistent scaffolds (ToD View)

Woven ELAC Scaffold

B

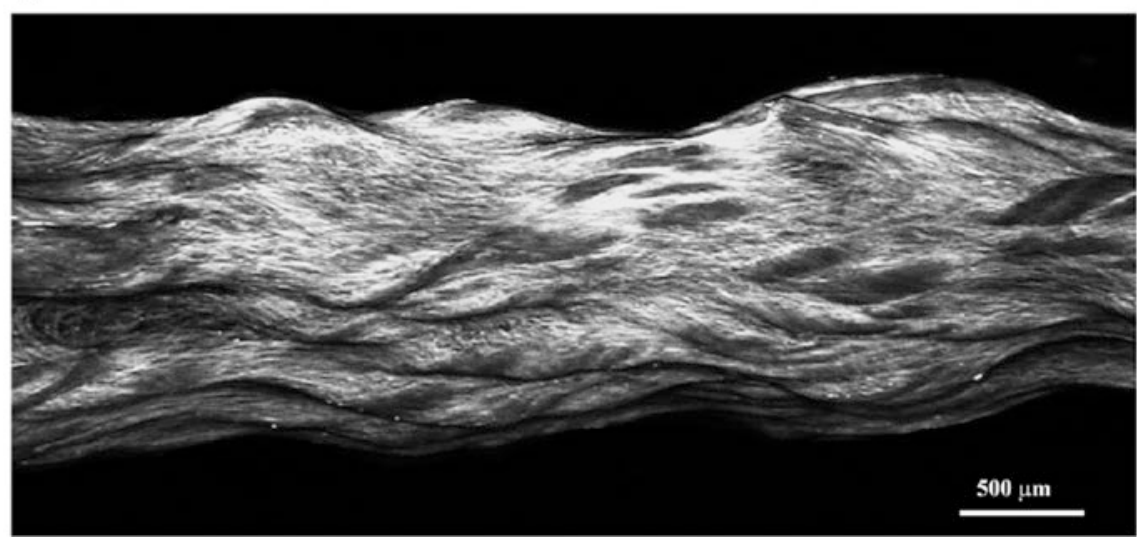


4 Fig. 17.5 A Schematic of the rotating electrode electrochemical alignment device (a). The main parts of the device are: power supply for providing voltage for the electrochemical cell, the syringe pump, rotating electrodes wheel and collection spool. Compensated polarized image in the top left inset demonstrates the collagen molecules to be aligned parallel to the longer axis of the thread as manifested by the blue color. Closely packed and aligned topography of the fiber surface is evident from the electron microscopy image. $\mathbf{b}$ Collagen fiber made by a rotating electrode electrochemical aligning device (REEAD), $\mathbf{c}$ yarn made by twisting three collagen threads, $\mathbf{d}$ pin-setup for weaving the collagen scaffold, $\mathbf{e}$ the resulting woven collagen scaffold, $\mathbf{f}$ and two scaffolds to demonstrate the consistency of fabrication. B A macrograph of cell-seeded scaffold where the cellular F-actin cytoskeleton is labeled. Cells have profusely covered the entire scaffold with elongated morphology. Reprinted from Ref. [67]. Copyright 2014 WILEY-VCH Verlag GmbH \& Co. KGaA, Weinheim. With permission from John Wiley and Sons

scaffolds in T/L TE is still limited and has not yet been compared with other materials produced e.g. by electrospinning, as discussed on previous section, or decellularized tendon-derived matrix [93], which share the same basic composition of ELAC scaffolds. This lack of versatility may reveal critical in case of failure at any developmental stage because it restricts the approaches that can be followed to solve possible drawbacks.

\subsection{Conclusions}

In this chapter, it was presented the important role of tendon and ligament tissues in articular biomechanics and their unique hierarchical organization. Injuries associated to these tissues are a common problem and a leading cause of joint disability affecting the population in general, consequently influencing their quality of life.

The current conventional techniques to manage this problematic, in particular in knee ligaments and tendons, were presented and discussed. In spite of the reduction of the symptoms, i.e. the pain control and the reduction of the inflammation and swelling, the mid and long term outcomes may progress to knee OA and/or T/L weakening. Moreover, due to the limitations of nonsurgical approaches, the resource to surgery remains the most recurrent option to reduce the symptoms and provide some short to mid term quality of life to patients.

In this sense, TE may become the most promising alternative therapy to achieve a complete regeneration of the damaged tissue, recovering its native biomechanical functionality. Nevertheless, despite the remarkable progresses made in the last few years, there are still significant challenges to overcome in this field. It spans from the establishment of cell sourcing and differentiation protocols, to the refinement of 3D scaffold designs that can simultaneously mimic the hierarchical and anisotropic architecture of $\mathrm{T} / \mathrm{L}$ and match their biomechanical behavior.

The continuous developments and promising outcomes are expected to bring $\mathrm{T} / \mathrm{L}$ TE strategies a step closer to clinic practice in a near future. 
Acknowledgments The authors wish to acknowledge the financial support of the Portuguese Foundation for Science and Technology for the post-doctoral grant (SFRH/BPD/111729/2015) and for the projects Recognize (UTAP-ICDT/CTM-BIO/0023/2014) and POCI-01-0145-FEDER-007038.

\section{References}

1. Frank CB (2004) Ligament structure, physiology and function. J Musculoskelet Neuronal Interact 4(2):199-201

2. Weintraub W (2003) The nature of tendons and ligaments. In Tendon and ligament healing: a new approach to sports and overuse injury. P. Publications, pp 5-24

3. Thorpe CT et al (2015) Chapter 1-Tendon physiology and mechanical behavior: structurefunction relationships. In: Gomes ME, Reis RL, Rodrigues MT (eds) Tendon regeneration. Academic Press, Boston, pp 3-39

4. Woo SL-Y et al (2007) Chapter 9-Functional tissue engineering of ligament and tendon injuries. In: Mao JJ et al (eds) Transitional approaches in tissue engineering and regenerative medicine. Artech House Publishers, Norwood

5. Hsu S-L, Liang R, Woo SL (2010) Functional tissue engineering of ligament healing. BMC Sports Science, Medicine and Rehabilitation, pp 2-12

6. Costa-Almeida R et al (2015) Tendon stem cell niche. In: Turksen K (ed) Tissue engineering and stem cell niche. Springer, Berlin, pp 221-244

7. Wang JHC (2006) Mechanobiology of tendon. J Biomech 39(9):1563-1582

8. Andrades JA et al (2011) Chapter 5-Skeletal regeneration by mesenchymal stem cells: what else? In: Eberli D (ed) Regenerative medicine and tissue engineering - cells and biomaterials. InTech, Morn Hill

9. Ghosh KM, Deehan DJ (2010) Soft tissue knee injuries. Surg Oxf Int Ed 28(10):494-501

10. Cowin SC, Doty SB (2007) The constituents of tendons and ligaments. In: Cowin SC, Doty SB (eds) Tissue mechanics. Springer, New York, p 562

11. Sharma P, Maffulli N (2006) Biology of tendon injury: healing, modeling and remodeling. J Musculoskelet Neuronal Interact 6(2):181-190

12. Riggin CN, Morris TR, Soslowsky LJ (2015) Chapter 5-Tendinopathy II: etiology, pathology, and healing of tendon injury and disease. In: Gomes ME, Reis RL, Rodrigues MT (eds) Tendon regeneration. Academic Press, Boston, pp 149-183

13. Ackermann PW (2015) Chapter 4-Tendinopathy I: understanding epidemiology, pathology, healing, and treatment. In: Gomes ME, Reis RL, Rodrigues MT (eds) Tendon regeneration. Academic Press, Boston, pp 113-147

14. Rodrigues MT, Reis RL, Gomes ME (2013) Engineering tendon and ligament tissues: present developments towards successful clinical products. J Tissue Eng Regen Med 7(9):673-686

15. Siegel L, Vandenakker-Albanese C, Siegel D (2012) Anterior cruciate ligament injuries: anatomy, physiology, biomechanics, and management. Clin J Sport Med 22(4):349-355

16. Kiapour AM, Murray MM (2014) Basic science of anterior cruciate ligament injury and repair. Bone Joint Res 3(2):20-31

17. Mascarenhas $\mathrm{R}$ et al (2012) Bone-patellar tendon-bone autograft versus hamstring autograft anterior cruciate ligament reconstruction in the young athlete: a retrospective matched analysis with 2-10 year follow-up. Knee Surg Sports Traumatol Arthrosc 20:1520-1527

18. Øiestad BE, Holm I, Engebretsen L, Aune AK, Gunderson R, Risberg MA (2013) The prevalence of patellofemoral osteoarthritis 12 years after anterior cruciate ligament reconstruction. Knee Surg Sports Traumatol Arthrosc 21(4):942-949

19. Liu Z-T et al (2010) Four-strand hamstring tendon autograft versus LARS artificial ligament for anterior cruciate ligament reconstruction. Int Orthop 34(1):45-49

20. Batty LM et al (2015) Synthetic devices for reconstructive surgery of the cruciate ligaments: a systematic review. Arthroscopy 31(5):957-968 
21. Tiefenboeck TM et al (2015) Clinical and functional outcome after anterior cruciate ligament reconstruction using the LARS ${ }^{\mathrm{TM}}$ system at a minimum follow-up of 10 years. Knee 22 (6):565-568

22. Chen J et al (2009) Scaffolds for tendon and ligament repair: review of the efficacy of commercial products. Expert Rev Med Devices 6(1):61-73

23. Yannas IV (2001) Tissue and organ regeneration in adults. Springer, New York

24. Czaplewski SK et al (2014) Tenogenic differentiation of human induced pluripotent stem cell-derived mesenchymal stem cells dictated by properties of braided submicron fibrous scaffolds. Biomaterials 35(25):6907-6917

25. Steinert AF et al (2011) Mesenchymal stem cell characteristics of human anterior cruciate ligament outgrowth cells. Tissue Eng Part A 17(9-10):1375-1388

26. Bi Y et al (2007) Identification of tendon stem/progenitor cells and the role of the extracellular matrix in their niche. Nat Med 13(10):1219-1227

27. Chen X et al (2009) Stepwise differentiation of human embryonic stem cells promotes tendon regeneration by secreting fetal tendon matrix and differentiation factors. Stem Cells 27 (6): 1276-1287

28. Xu W et al (2013) Human iPSC-derived neural crest stem cells promote tendon repair in a rat patellar tendon window defect model. Tissue Eng Part A 19(21-22):2439-2451

29. Otabe $\mathrm{K}$ et al (2015) The transcription factor Mohawk controls tenogenic differentiation of bone marrow mesenchymal stem cells in vitro and in vivo. J Orthop Res 33(1):1-8

30. Tan S-L et al (2015) Identification of pathways mediating growth differentiation factor5-induced tenogenic differentiation in human bone marrow stromal cells. PLoS ONE 10(11):e0140869

31. Urdzikova LM, Sedlacek R, Suchy T, Amemori T, Ruzicka J, Lesny P, Havlas V, Jendelova P (2014) Human multipotent mesenchymal stem cells improve healing after collagenase tendon injury in the rat. Biomed Eng Online 13(42). doi:10.1186/1475-925X-13-42, http:// biomedical-engineering-online.biomedcentral.com/articles/10.1186/1475-925X-13-42

32. Gonçalves AI et al (2014) Understanding the role of growth factors in modulating stem cell tenogenesis. PLoS ONE 8(12):e83734

33. de Mattos Carvalho A et al (2011) Use of adipose tissue-derived mesenchymal stem cells for experimental tendinitis therapy in equines. J Equine Vet Sci 31(1):26-34

34. Molloy T, Wang Y, Murrell GAC (2003) The roles of growth factors in tendon and ligament healing. Sports Med 33(5):381-394

35. Temenoff JS, Mikos AG (2000) Review: tissue engineering for regeneration of articular cartilage. Biomaterials 21(5):431-440

36. Rizzello $\mathrm{G}$ et al (2012) Growth factors and stem cells for the management of anterior cruciate ligament tears. Open Orthop J 6:525-530

37. Klein MB et al (2002) Flexor tendon healing in vitro: effects of TGF- $\beta$ on tendon cell collagen production. J Hand Surg 27(4):615-620

38. Chan BP et al (2000) Effects of basic fibroblast growth factor (bFGF) on early stages of tendon healing: a rat patellar tendon model. Acta Orthop Scand 71(5):513-518

39. Sahoo S, Toh SL, Goh JCH (2010) A bFGF-releasing silk/PLGA-based biohybrid scaffold for ligament/tendon tissue engineering using mesenchymal progenitor cells. Biomaterials 31 (11):2990-2998

40. Murray MM et al (2007) Enhanced histologic repair in a central wound in the anterior cruciate ligament with a collagen-platelet-rich plasma scaffold. J Orthop Res 25(8):1007-1017

41. Murray $\mathrm{M}$ et al (2006) Use of a collagen-platelet rich plasma scaffold to stimulate healing of a central defect in the canine ACL. J Orthop Res 24(4):820-830

42. Ricchetti ET et al (2012) Scaffold devices for rotator cuff repair. J Shoulder Elbow Surg 21 (2):251-265

43. Dunn MG et al (1995) Development of fibroblast-seeded ligament analogs for ACL reconstruction. J Biomed Mater Res 29(11):1363-1371

44. Bellincampi LD et al (1998) Viability of fibroblast-seeded ligament analogs after autogenous implantation. J Orthop Res 16(4):414-420 
45. Weadock K et al (1995) Physical crosslinking of collagen fibers: comparison of ultraviolet irradiation and dehydrothermal treatment. J Biomed Mater Res 29(11):1373-1379

46. Gurkan UA et al (2010) Comparison of morphology, orientation, and migration of tendon derived fibroblasts and bone marrow stromal cells on electrochemically aligned collagen constructs. J Biomed Mater Res, Part A 94A(4):1070-1079

47. Altman GH et al (2003) Silk-based biomaterials. Biomaterials 24(3):401-416

48. Vepari C, Kaplan DL (2007) Silk as a biomaterial. Prog Polym Sci 32(8-9):991-1007

49. Chen JL et al (2010) Efficacy of hESC-MSCs in knitted silk-collagen scaffold for tendon tissue engineering and their roles. Biomaterials 31(36):9438-9451

50. Chen X et al (2014) Scleraxis-overexpressed human embryonic stem cell-derived mesenchymal stem cells for tendon tissue engineering with knitted silk-collagen scaffold. Tissue Eng Part A 20(11-12):1583-1592

51. Chen $J$ et al (2003) Human bone marrow stromal cell and ligament fibroblast responses on RGD-modified silk fibers. J Biomed Mater Res, Part A 67(2):559-570

52. Majima $T$ et al (2007) Chitosan-based hyaluronan hybrid polymer fibre scaffold for ligament and tendon tissue engineering. Proc Inst Mech Eng Part H 221(5):537-546

53. Moffat KL et al (2009) Novel nanofiber-based scaffold for rotator cuff repair and augmentation. Tissue Eng Part A 15(1):115-126

54. Fan $\mathrm{H}$ et al (2009) Anterior cruciate ligament regeneration using mesenchymal stem cells and silk scaffold in large animal model. Biomaterials 30(28):4967-4977

55. Laitinen $O$ et al (1992) Mechanical properties of biodegradable ligament augmentation device of poly(L-lactide) in vitro and in vivo. Biomaterials 13(14):1012-1016

56. Cooper JA et al (2007) Biomimetic tissue-engineered anterior cruciate ligament replacement. Proc Natl Acad Sci USA 104(9):3049-3054

57. Freeman JW, Woods MD, Laurencin CT (2007) Tissue engineering of the anterior cruciate ligament using a braid-twist scaffold design. J Biomech 40(9):2029-2036

58. Petrigliano FA et al (2007) The effects of local bFGF release and uniaxial strain on cellular adaptation and gene expression in a 3D environment: implications for ligament tissue engineering. Tissue Eng 13(11):2721-2731

59. Leong NL et al (2015) Evaluation of polycaprolactone Scaffold with basic fibroblast growth factor and fibroblasts in an athymic rat model for anterior cruciate ligament reconstruction. Tissue Eng Part A 21(11-12):1859-1868

60. Xu Y et al (2013) Fabrication of electrospun poly(L-lactide-co- $\varepsilon$-caprolactone)/collagen nanoyarn network as a novel, three-dimensional, macroporous, aligned scaffold for tendon tissue engineering. Tissue Eng Part C Methods 19(12):925-936

61. Xu Y et al (2014) The effect of mechanical stimulation on the maturation of TDSCs-poly(Llactide-co-e-caprolactone)/collagen scaffold constructs for tendon tissue engineering. Biomaterials 35(9):2760-2772

62. Leung $M$ et al (2013) Tenogenic differentiation of human bone marrow stem cells via a combinatory effect of aligned chitosan-poly-caprolactone nanofibers and TGF-[small beta]3 J Mater Chem B 1(47):6516-6524

63. Domingues RMA et al (2016) Enhancing the biomechanical performance of anisotropic nanofibrous scaffolds in tendon tissue engineering: reinforcement with cellulose nanocrystals. Adv Healthc Mater. doi:10.1002/adhm.201501048

64. Wang L et al (2015) Nanofiber yarn/hydrogel core-shell scaffolds mimicking native skeletal muscle tissue for guiding 3D myoblast alignment, elongation, and differentiation. ACS Nano 9 (9):9167-9179

65. Shuakat MN, Lin T (2014) Recent developments in electrospinning of nanofiber yarns. J Nanosci Nanotechnol 14(2):1389-1408

66. Zhang $\mathrm{C}$ et al (2015) Well-aligned chitosan-based ultrafine fibers committed teno-lineage differentiation of human induced pluripotent stem cells for Achilles tendon regeneration. Biomaterials 53:716-730

67. Younesi M et al (2014) Tenogenic induction of human MSCs by anisotropically aligned collagen biotextiles. Adv Funct Mater 24(36):5762-5770 
68. Domingues RMA, Gomes ME, Reis RL (2014) The potential of cellulose nanocrystals in tissue engineering strategies. Biomacromolecules 15(7):2327-2346

69. Nivison-Smith L, Weiss AS (2012) Alignment of human vascular smooth muscle cells on parallel electrospun synthetic elastin fibers. J Biomed Mater Res, Part A 100A(1):155-161

70. Spanoudes K et al (2014) The biophysical, biochemical, and biological toolbox for tenogenic phenotype maintenance in vitro. Trends Biotechnol 32(9):474-482

71. Yin $\mathrm{Z}$ et al (2010) The regulation of tendon stem cell differentiation by the alignment of nanofibers. Biomaterials 31(8):2163-2175

72. Zhou C et al (2013) Electrospun bio-nanocomposite scaffolds for bone tissue engineering by cellulose nanocrystals reinforcing maleic anhydride grafted PLA. ACS Appl Mater Interfaces 5(9):3847-3854

73. Abbah SA, Spanoudes K, O’Brien T, Pandit A, Zeugolis DI (2014) Assessment of stem cell carriers for tendon tissue engineering in pre-clinical models. Stem Cell Res Ther 5(38). doi:10. 1186/scrt426, http://stemcellres.biomedcentral.com/articles/10.1186/scrt426

74. Barber JG et al (2011) Braided nanofibrous scaffold for tendon and ligament tissue engineering. Tissue Eng Part A 19(11-12):1265-1274

75. Liu W et al (2015) Generation of electrospun nanofibers with controllable degrees of crimping through a simple, plasticizer-based treatment. Adv Mater 27(16):2583-2588

76. Surrao DC et al (2012) A crimp-like microarchitecture improves tissue production in fibrous ligament scaffolds in response to mechanical stimuli. Acta Biomater 8(10):3704-3713

77. Chen F, Hayami JWS, Amsden BG (2014) Electrospun poly(L-lactide-co-acryloyl carbonate) fiber scaffolds with a mechanically stable crimp structure for ligament tissue engineering. Biomacromolecules 15(5):1593-1601

78. Lomas AJ et al (2015) The past, present and future in scaffold-based tendon treatments. Adv Drug Deliv Rev 85:257-277

79. LaCroix AS et al (2013) Relationship between tendon stiffness and failure: a metaanalysis. J Appl Physiol 115(1):43-51

80. Domingues RMA et al (2015) Chapter 10 - Fabrication of hierarchical and biomimetic fibrous structures to support the regeneration of tendon tissues. In: Gomes ME, Reis RL, Rodrigues MT (eds) Tendon regeneration. Academic Press, Boston, pp 259-280

81. Pauly HM et al (2016) Mechanical properties and cellular response of novel electrospun nanofibers for ligament tissue engineering: effects of orientation and geometry. J Mech Behav Biomed Mater 61:258-270

82. Bosworth LA et al (2013) Investigation of 2D and 3D electrospun scaffolds intended for tendon repair. J Mater Sci - Mater Med 24(6):1605-1614

83. Mouthuy P-A et al (2015) Fabrication of continuous electrospun filaments with potential for use as medical fibres. Biofabrication 7(2):025006

84. Yang G et al (2016) Multilayered polycaprolactone/gelatin fiber-hydrogel composite for tendon tissue engineering. Acta Biomater 35:68-76

85. Aibibu D et al (2016) Textile cell-free scaffolds for in situ tissue engineering applications. J Mater Sci - Mater Med 27:63

86. Cheng $X$ et al (2008) An electrochemical fabrication process for the assembly of anisotropically oriented collagen bundles. Biomaterials 29(22):3278-3288

87. Uquillas JA, Kishore V, Akkus A (2011) Effects of phosphate-buffered saline concentration and incubation time on the mechanical and structural properties of electrochemically aligned collagen threads. Biomed Mater 6(3):035008

88. Uquillas JA, Kishore V, Akkus O (2012) Genipin crosslinking elevates the strength of electrochemically aligned collagen to the level of tendons. J Mech Behav Biomed Mater 15:176-189

89. Kishore V et al (2011) Incorporation of a decorin biomimetic enhances the mechanical properties of electrochemically aligned collagen threads. Acta Biomater 7(6):2428-2436

90. Kishore V et al (2012) Tenogenic differentiation of human MSCs induced by the topography of electrochemically aligned collagen threads. Biomaterials 33(7):2137-2144 
91. Kishore V et al (2012) In vivo response to electrochemically aligned collagen bioscaffolds. J Biomed Mater Res B Appl Biomater 100B(2):400-408

92. Islam A et al (2015) Biomechanical evaluation of a novel suturing scheme for grafting load-bearing collagen scaffolds for rotator cuff repair. Clin Biomech 30(7):669-675

93. Cheng CW, Solorio LD, Alsberg E (2014) Decellularized tissue and cell-derived extracellular matrices as scaffolds for orthopaedic tissue engineering. Biotechnol Adv 32(2):462-484 